\title{
Review: ear pain and a cloudy, bulging, or distinctly immobile tympanic membrane appear to help diagnose acute otitis media in children
}

Rothman R, Owens, Simel DL. Does this child have acute otitis media? JAMA 2003;290:1633-40.

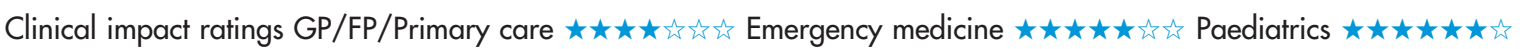

In children, how accurate are symptoms and signs for diagnosing acute otitis media (AOM)?

\section{METHODS}

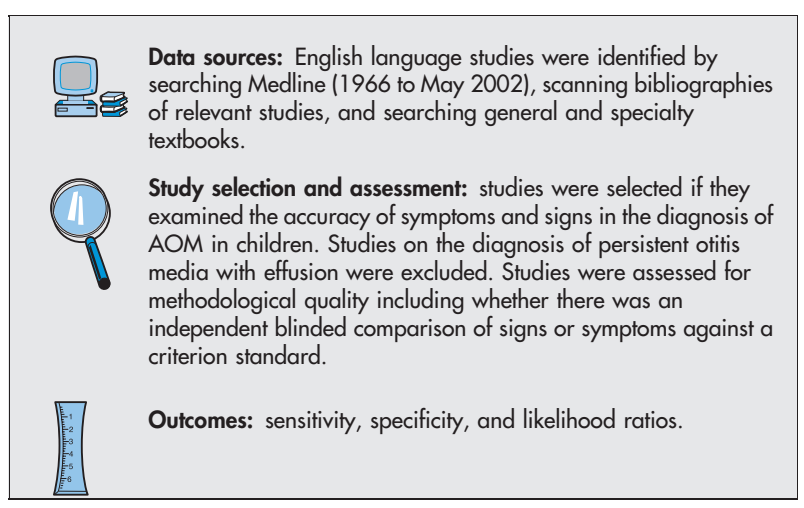

\section{MAIN RESULTS}

6 studies met the selection criteria. 1 study ( 43 children) examined precision and found only a fair overall agreement (kappa statistic 0.30 ) on diagnosis of AOM between paediatric residents and otolaryngologists. 4 studies (total 965 children; age range 0 to $15 \mathrm{y}$ ) examined the accuracy of symptoms; in these studies, clinical diagnosis was used as the criterion standard. Using clinical diagnosis as the criterion standard, the presence of ear pain and parental suspicion of AOM may be useful for diagnosing AOM. 1 study (2911 children; age range 6 mo to $2.5 \mathrm{y}$ ) examined the accuracy of signs; in this study, tympanocentesis was used as the criterion standard. This study reported the accuracy of signs on pneumatic otoscopy for diagnosing AOM in children (table).

\section{CONCLUSION}

In children, ear pain and a cloudy, bulging, or distinctly immobile tympanic membrane appear to be helpful for diagnosing acute otitis media.

For correspondence: Dr R Rothman, Vanderbilt University Medical Center, Nashville, TN, USA. russell.rothman@vanderbilt.edu

Source of funding: Robert Wood Johnson Clinical Scholars Program.

\section{Commentary}

To decrease the development of bacterial resistance to antimicrobials, their use should be minimised. To achieve this goal in paediatric infections would require a decrease in use of antimicrobial therapy for otitis media. In their systematic review, Rothman et al analysed the data of the accuracy of symptoms and signs as diagnostic tests for acute otitis media. They agreed with the conclusions of the separate surveys that no symptoms exist that are independently useful in diagnosing acute otitis media in children. Thus, more emphasis should be put on the diagnostics of otitis media. Tympanometry is a reliable diagnostic aid' and is especially useful now as we have given up doing tympanocentesis in AOM. An external diagnostic tool is needed and pneumatic otoscopy, although moderately accurate for AOM (table), may be difficult to teach.

One reason why physicians prescribe antimicrobials for children is their assumption of parental expectations of antibiotics. Physicians often overestimate the parents' expectations, and change in parental expectations does not change physicians' prescribing behaviour. ${ }^{2}{ }^{3}$ Providing a contingency plan to parents who expect antibiotics for their children has been shown to improve parental satisfaction without antimicrobial prescription. ${ }^{2}$ Thus, parents should not be blamed, and we physicians urgently need to stop over prescribing antimicrobials.

Matti Uhari, MD, MSc University of Oulu Oulu, Finland

1 Koivunen $\mathrm{P}$, Alho OP, Uhari $\mathrm{M}$, et al. Minitympanometry in detecting middle ear fluid. $J$ Pediatr 1997;131:419-22.

2 Mangione-Smith R, McGlynn EA, Elliott MN, et al. Parent expectations for antibiotics, physician-parent communication, and satisfaction. Arch Pediatr Adolesc Med 2001; 155:800-6.

3 Wheeler JG, Fair M, Simpson PM, et al. Impact of a waiting room videotape message on parent attitudes toward pediatric antibiotic use. Pediatrics 2001;108:591-6.

Accuracy of signs on pneumatic otoscopy for diagnosing acute otitis media in children*

\begin{tabular}{llll}
\hline Sign & Result & Unadjusted + LR & Adjusted +LRT \\
\hline Colour & Cloudy colour & 11 & 34 \\
& Distinctly red colour & 2.6 & 8.4 \\
& Slightly red colour & 0.4 & 1.4 \\
& Normal colour & 0.1 & 0.2 \\
Position & Bulging position & 20 & 51 \\
& Retracted position & 1.3 & 3.5 \\
& Normal position & 0.4 & 0.5 \\
Mobility & Distinctly impaired mobility & 8.4 & 31 \\
& Slightly impaired mobility & 1.1 & 4.0 \\
& Normal mobility & 0.04 & 0.2 \\
\hline \multirow{2}{*}{ *LR defined in glossary. } & & \\
†+LRs adjusted to correct for verification bias. & \\
\hline
\end{tabular}

